\title{
The timeline and risk factors of clinical progression of COVID-19 in Shenzhen, China
}

\author{
Fang Wang ${ }^{1 \dagger}$, Mengyuan $\mathrm{Qu}^{2 \dagger}$, Xuan Zhou ${ }^{1 \dagger}$, Kai Zhao ${ }^{2 \dagger}$, Changxiang Lai ${ }^{1}$, Qiyuan Tang ${ }^{1}$, Wenjie Xian ${ }^{1}$,
} Ruikun Chen', Xuan $\mathrm{Li}^{1}$, Zhiyu Li ${ }^{1}$, Qing He ${ }^{1 *}$ and Lei Liu ${ }^{1 *}$

\begin{abstract}
Background: The novel coronavirus disease 2019 (COVID-19) broke out globally. Early prediction of the clinical progression was essential but still unclear. We aimed to evaluate the timeline of COVID-19 development and analyze risk factors of disease progression.

Methods: In this retrospective study, we included 333 patients with laboratory-confirmed COVID-19 infection hospitalized in the Third People's Hospital of Shenzhen from 10 January to 10 February 2020. Epidemiological feature, clinical records, laboratory and radiology manifestations were collected and analyzed. 323 patients with mild-moderate symptoms on admission were observed to determine whether they exacerbated to severe-critically ill conditions (progressive group) or not (stable group). We used logistic regression to identify the risk factors associated with clinical progression.

Results: Of all the 333 patients, 70 (21.0\%) patients progressed into severe-critically ill conditions during hospitalization and assigned to the progressive group, 253 (76.0\%) patients belonged to the stable group, another 10 patients were severe before admission. we found that the clinical features of aged over $40(3.80[1.72,8.52])$, males $(2.21[1.20$, $4.07])$, with comorbidities $(1.78[1.13,2.81])$ certain exposure history $(0.38[0.20,0.71])$, abnormal radiology manifestations $(3.56[1.13,11.40])$, low level of T lymphocytes $(0.99[0.997,0.999])$, high level of NLR $(0.99[0.97,1.01])$, IL-6 (1.05 $[1.03,1.07])$ and $\operatorname{CRP}(1.67[1.12,2.47])$ were the risk factors of disease progression by logistic regression.
\end{abstract}

Conclusions: The potential risk factors of males, older age, with comorbidities, low T lymphocyte level and high level of NLR, CRP, IL- 6 can help to predict clinical progression of COVID-19 at an early stage.

Keywords: COVID-19, Pneumonia, Clinical progression, Risk factor, Retrospective analysis

\section{Background}

Since December 2019, a novel coronavirus disease (formerly known as 2019-nCoV and now renamed COVID19) had rapidly spread throughout China, leading to a global outbreak and causing considerable public

\footnotetext{
*Correspondence: a549719965@163.com; liuleiszsdsrmyy@163.com ${ }^{\dagger}$ Fang Wang, Mengyuan Qu, Xuan Zhou and Kai Zhao contribute equally to the article as the co-first authors

1 Department of Hepatology, Shenzhen Third People's Hospital, National Clinical Research Center for Infectious Disease, The Second Affiliated Hospital, School of Medicine, Southern University of Science and Technology, 29 Bulan Road, Shenzhen 518112, Guangdong, China Full list of author information is available at the end of the article
}

health concern $[1,2]$. Until February 28th, 2020, the latest update from China's National Health Commission reported there had been 78959 confirmed cases of the infection. Despite the lower case fatality rate, COVID19 has so far resulted in more deaths (2791) than severe acute respiratory syndrome (SARS) and middle east respiratory syndrome (MERS) combined (1632) [3].

Despite the increasing confirmed cases updated daily, the clinical investigation of affected patients was limited. In an early study in Wuhan of 138 hospitalized patients, the mortality was $4.3 \%$ and $26 \%$ of patients received intensive care unit (ICU) care [4]. However, another research from Zhejiang province reported that of the

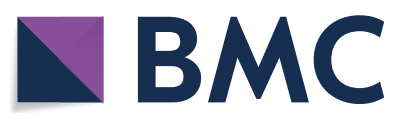

c) The Author(s) 2020. This article is licensed under a Creative Commons Attribution 4.0 International License, which permits use, sharing, adaptation, distribution and reproduction in any medium or format, as long as you give appropriate credit to the original author(s) and the source, provide a link to the Creative Commons licence, and indicate if changes were made. The images or other third party material in this article are included in the article's Creative Commons licence, unless indicated otherwise in a credit line to the material. If material is not included in the article's Creative Commons licence and your intended use is not permitted by statutory regulation or exceeds the permitted use, you will need to obtain permission directly from the copyright holder. To view a copy of this licence, visit http://creativeco mmons.org/licenses/by/4.0/. The Creative Commons Public Domain Dedication waiver (http://creativecommons.org/publicdomain/ zero/1.0/) applies to the data made available in this article, unless otherwise stated in a credit line to the data. 
62 patients studied, the symptoms were relatively mild compared with Wuhan's situation, only one was admitted to ICU, and no patients died during the study [5]. The significant contrast between those two made us want to explore further. Conclusions drawn from Wuhan alone might be biased and could not be representative of overall conditions due to its overwhelmingly rapid transmission and limited medical resources at the very beginning of the outbreak [6]. Thus, infected cases from regions outside Hubei can better inform the disease's epidemiological and clinical characteristics.

Shenzhen, located near Hong Kong, served as a Special Economic Zone in southern China, has a large population density and high mobility, and therefore faces a comparatively high epidemics danger and transmission risk. The third people's Hospital of Shenzhen is the only designated hospital which is authorized to admit all patients confirmed with COVID-19 in Shenzhen. In this study, we aimed to retrospectively describe the clinical features and laboratory findings of COVID-19 and also focused on searching for possible risk factors for clinical progression of severe patients in Shenzhen, and hopefully, providing valuable experience of patient management and stratification for other metropolises overseas.

\section{Materials and methods}

\section{Study design and participants}

It is a retrospective, single-center case series of the 333 hospitalized patients diagnosed with COVID-19 in the third people's hospital of Shenzhen. We recruited all the confirmed patients whose admission date was from January 10, 2020 to February 10, 2020. All 333 COVID-19 patients were classified as mild, moderate, severe or critically ill category at admission. The diagnostic standard and classifying criteria of COVID-19 were based on the interim guidance from the WHO [7].

Two cohorts were generated in our research: 323 patients with mild-moderate symptoms on admission were observed for at least 18 days to determine whether they exacerbated to severe-critically ill conditions (progressive group) or not (stable group). In addition, we analyze the other 10 patients left who were severe-critically ill from the beginning of admission separately.

\section{Laboratory confirmation and data collection}

Suspected cases were confirmed by positive real-time PCR assay for SARS-CoV-2. Pharyngeal swab specimens were collected on admission and may test several times for the COVID-19 confirmation. Other laboratory assessments included the whole blood count, electrolytes, coagulation test, liver and renal function, myocardial zymogram, C-reactive protein (CRP), procalcitonin, lactate dehydrogenase (LDH), Erythrocyte sedimentation rate (ESR), arterial O2/CO2 pressure and the like. Moreover, a typical chest computed tomography (CT) include multifocal bilateral ground-glass opacity with patchy consolidation. Massive consolidation with small pleural effusions and even "white lung" can be seen in severecritically ill COVID-19 pneumonia [8]. The blood samples and CT scan were acquired on admission.

We extracted the medical records of the patients with COVID-19 and collected all the detailed data upon admission, including the basic information, epidemiological feature, clinical characteristic, laboratory finding as well as chest $\mathrm{CT}$ imaging.

\section{Statistical analysis}

Continuous variables were described as the medians and interquartile ranges (IQR). Categorical variables were summarized as the frequencies and percentages in each category. Mann-Whitney test were applied to continuous variables, and Chi square test or Fisher's exact test were used for categorical variables. Univariate and multivariate logistic regression analysis were adopted to identify risk factors of disease progression, and the Mann-Whitney test was used. For comparisons, a two-sided $\alpha$ of less than 0.05 was considered statistically significant. Statistical analyses were conducted with SPSS software version 23.0.

\section{Results}

The epidemic trend and outline of the COVID-19

Located in the south of China with a population of 13.0 million, Shenzhen reported its first confirmed case on January, 19th 2020. Up until February 19th 2020, there were totally 417 cases confirmed according to the official reports. The epidemical trends of new cases, cumulative cases and remaining cases were shown in the Fig. 1a, newly confirmed cases per day reached the peak in around 12 days after first case report and the remaining cases started to decrease after about 20 days. Since February 18th, there were barely new cases added and the situation turned better.

To explore the timeline and disease progression of COVID-19, we focus on the 333 confirmed cases in the first month (admission date from Jan 10th to Feb 10th) (Fig. 1b). Most of the patients were admitted to hospital within 4 days after the disease onset, the median interval from disease onset to admission was 3 days (range 1-5 days) (Fig. 1c). In the 254 confirmed cases who had clear and credible information of exposure contacts to calculate the incubation period, the median of incubation period was 7 days (range 4-12 days). During the hospitalization, 70 (21.1\%) mild-moderate cases progressed to severe condition in the median 5 days (range 2-8 days), $23(6.9 \%)$ cases were admitted to ICU in median 2 days 

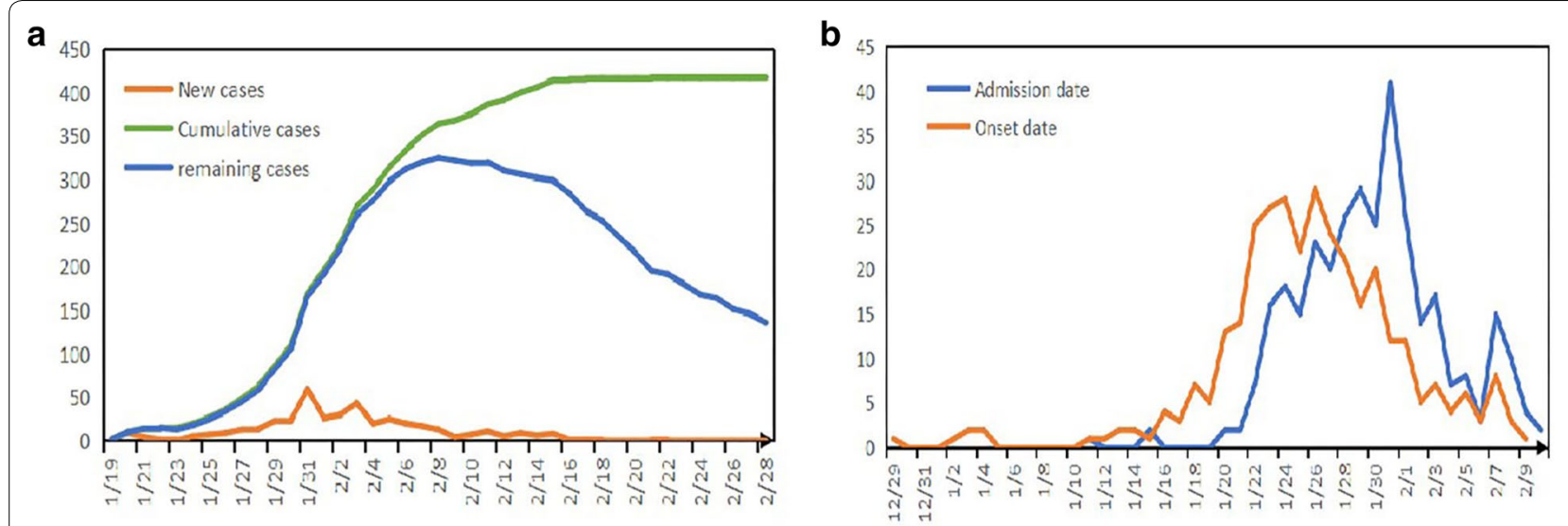

C

\section{Contact}

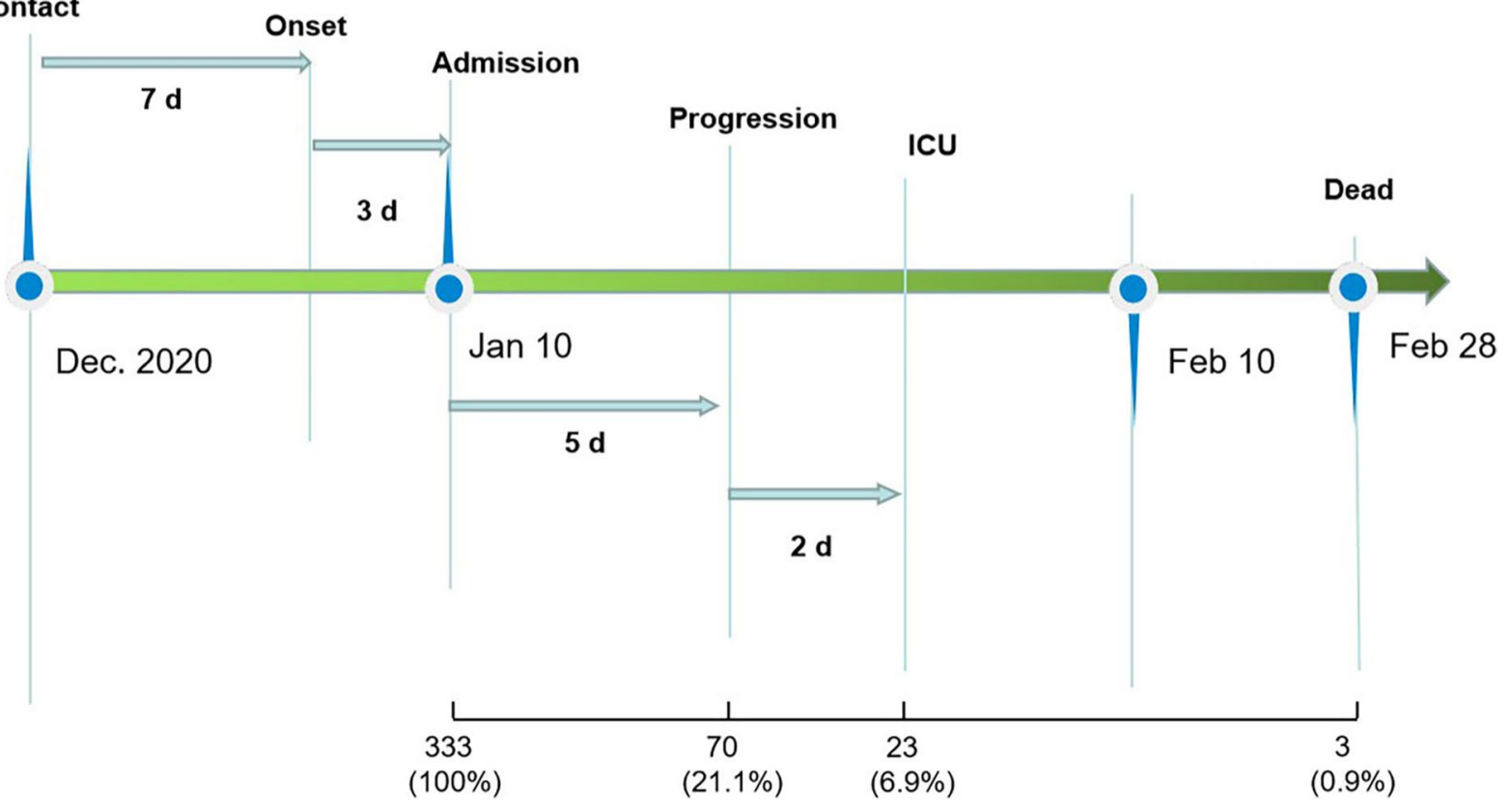

Fig. 1 The epidemic trend and timeline in a COVID-19-designated hospital. a The outbreak of COVID-19 in Shenzhen according to official data from Jan. 10 to Feb. 28. b The admission date and onset date in the designated hospital. c The timeline of COVID-19 cases in the first month of admission

(range 1-4 days) after progression and unfortunately 3 (0.9\%) patients died by the end of Feb 28th (Fig. 1c).

The baseline clinical characteristics of disease progression The median age of all the 323 patients was 46 years (IQR, $33-59$; range, 8 months to 86 years), the age range and proportions were shown in the Fig. 2a. A total of 333 patients were classified according to the criteria defined above. The proportion of patients with mild, moderate, severe and critical on admission were $7.5 \%$ (25/333), 89.5\% (298/333), $2.1 \%(7 / 333)$, and $0.9 \%(3 / 333)$, respectively. The spectrum of severity of diseases changed as disease progressed, 70 mild-moderate cases progressed to severe condition (progressive group), while 253 patients did not (stable group), and another 10 patients were severe from the beginning of admission (Fig. 2b).

Patients who later progressed to severe condition were more likely to have underlying comorbidities compared with the stable group $(42.8 \%$ vs $16.6 \%, \mathrm{P}<0.05)$. Of all, hypertension was the most common disease $(35,10.8 \%)$, followed by diabetes $(5.0 \%)$, heart diseases $(4.0 \%)$, pulmonary disease $(2.8 \%)$, liver diseases $(2.5 \%)$, malignancy (0.9\%), cerebrovascular disease $(0.3 \%)$ and other conditions (2.1\%) (Fig. 2c). 

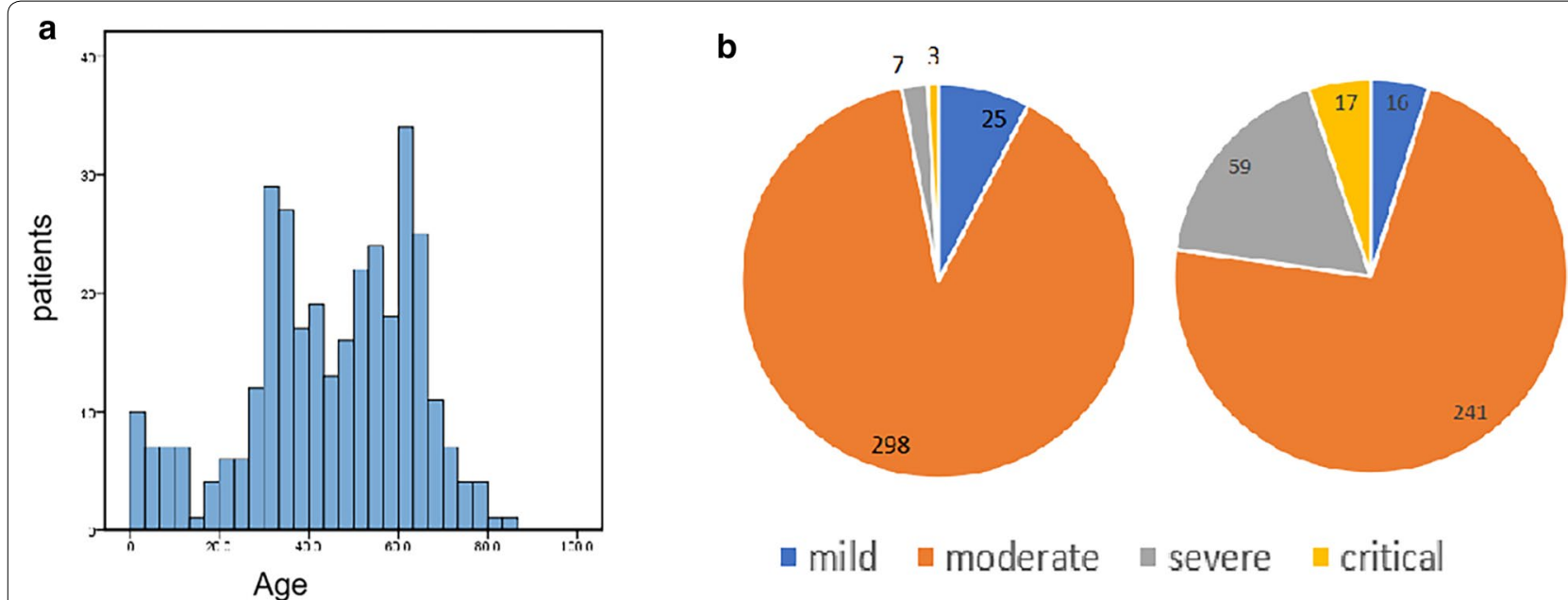

\section{$\square$ mild $\square$ moderate $\square$ severe $\square$ critical}

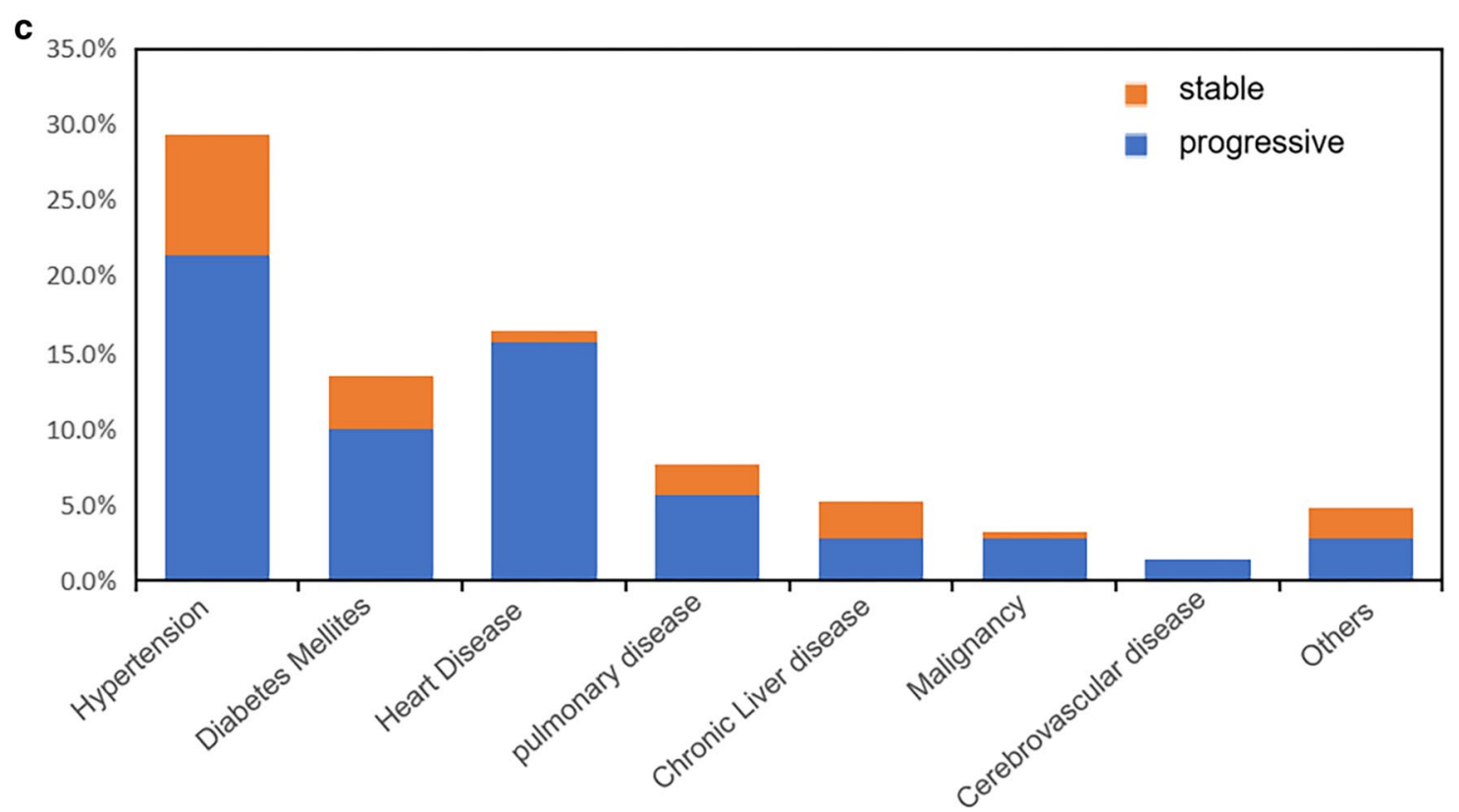

Fig. 2 The distribution of age, symptom and baseline clinical characteristics. a The distribution of age in the COVID-19 patients; $\mathbf{b}$ The proportion of Clinical Severity of Confirmed COVID-19 Pneumonia on the admission (Left) and progressed period (Right). $\mathbf{c}$ The proportion in the progressed and stable patients

\section{The clinical characteristics of the progressive and stable} groups

As shown in Table 1, compared with the stable group, the progressive group was significantly older $(P<0.001)$, there were no one under 18 years and patients over 65 years made up an evidently larger proportion (21.4\%) in this group. More than half of all patients $(169,52.3 \%)$ were females, however, apparently more men (64.3\%) ended up in progressive situation. Of all, 173 (53.6\%) patients had an exposure history related to Wuhan and 90 (27.9\%) cases were connected with other cities in Hubei province except Wuhan. Around 167 (51.7\%) patients lived in Shenzhen but had outside contacts with confirmed or suspected infections or experienced a short term trip outside, whereas only 11 (3.4\%) patients claimed no obvious exposure history. None of them were hospital-related transmission.

The most prevalent symptom was fever before admission $(248,76.8 \%)$ and it was almost comparable between two groups $(\mathrm{P}=0.154)$. Nearly half of patients were presented with pneumonia symptoms and systemic manifestations, including cough $(49.5 \%)$, expectoration (22.9\%), fatigue or myalgia (21.4\%), anorexia (12.4\%), dizziness $(8.0 \%)$, chest tightness $(5.0 \%)$, dyspnea $(2.8 \%)$, 
Table 1 Clinic characteristics and outlines of 323 patients infected with COVID-19 on admission

\begin{tabular}{|c|c|c|c|c|}
\hline Characteristics & Total $(\mathrm{N}=323)$ & Progressive $(\mathrm{N}=70)$ & Stable $(\mathrm{N}=253)$ & $P$ value \\
\hline Median age (years) & $46.0(33.0-59.0)$ & $59.5(49.0-64.0)$ & $41.0(32.0-56.0)$ & $<0.001$ \\
\hline Age groups (years): & & & & $<0.001$ \\
\hline$\leq 18$ & $35(10.8 \%)$ & $0(0 \%)$ & $35(13.9 \%)$ & \\
\hline $19-40$ & $106(32.8 \%)$ & $9(12.9 \%)$ & $97(38.3 \%)$ & \\
\hline $41-65$ & $150(32.5 \%)$ & $46(65.7 \%)$ & $104(41.1 \%)$ & \\
\hline$>65$ & $32(9.9 \%)$ & $15(21.4 \%)$ & $17(6.7 \%)$ & \\
\hline Sex: & & & & 0.002 \\
\hline Male & $154(47.7 \%)$ & $45(64.3 \%)$ & 109 (43.1\%) & \\
\hline Female & $169(52.3 \%)$ & $25(35.7 \%)$ & $144(56.9 \%)$ & \\
\hline Comorbidities & $72(22.3 \%)$ & $30(42.9 \%)$ & $42(16.6 \%)$ & $<0.001$ \\
\hline Incubation period & $7(4,12)$ & $7(3-12)$ & $7(4.0-11.5)$ & 0.994 \\
\hline From Onset to admission(days) & $3(1-5)$ & $4(2-6)$ & $3(1-5)$ & 0.006 \\
\hline Hospital stay (days) & $18(14-22)$ & $21(18.5-23)$ & $17.5(14.0-21.0)$ & 0.003 \\
\hline \multicolumn{5}{|l|}{ Exposure history: } \\
\hline Living in Shenzhen with outside contact & $167(51.7 \%)$ & $24(34.3 \%)$ & $143(56.5 \%)$ & $<0.001$ \\
\hline From outside to Shenzhen & $127(39.3 \%)$ & $33(47.1 \%)$ & $94(37.2 \%)$ & 0.130 \\
\hline No obvious exposure & $11(3.4 \%)$ & $5(7.1 \%)$ & $6(2.4 \%)$ & 0.051 \\
\hline Related to Wuhan & $173(53.6 \%)$ & $40(57.1 \%)$ & $133(52.6 \%)$ & 0.490 \\
\hline \multicolumn{5}{|l|}{ Signs and symptoms } \\
\hline Respiratory rate $>24$ breaths per min & $5(1.5 \%)$ & $3(4.3 \%)$ & $2(0.8 \%)$ & 0.036 \\
\hline Heart rate $>100$ per $m$ in & $62(19.2 \%)$ & $17(24.3 \%)$ & $45(17.8)$ & 0.222 \\
\hline Systolic pressure (mm Hg) & $125.0(115.0-136.0)$ & $132.0(123.5-140.5)$ & $122(112.0-133.0)$ & $<0.001$ \\
\hline Temperature on admission & $37.0(36.6-37.5)$ & $37.6(36.9-38.0)$ & $36.8(36.6-37.3)$ & $<0.001$ \\
\hline Fever & $248(76.8 \%)$ & $61(87.1 \%)$ & $187(73.9 \%)$ & 0.154 \\
\hline Cough & 160 (49.5\%) & $48(68.6 \%)$ & $112(44.3 \%)$ & $<0.001$ \\
\hline Expectoration & $74(22.9 \%$ & $30(42.9 \%)$ & $44(17.4 \%)$ & $<0.001$ \\
\hline Chest tightness & $16(5.0 \%)$ & $9(12.9 \%)$ & $7(2.8 \%)$ & $<0.001$ \\
\hline Dyspnea & $9(2.8 \%)$ & $5(7.1 \%)$ & $4(1.6 \%)$ & 0.012 \\
\hline Myalgia or fatigue & $69(21.4 \%)$ & $26(37.1 \%)$ & $43(17.0 \%$ & $<0.001$ \\
\hline Diarrhea & $24(7.4 \%)$ & $7(10.0 \%)$ & $17(6.7 \%)$ & 0.354 \\
\hline Headache & $26(8.0 \%)$ & $10(14.3 \%)$ & $16(6.3 \%)$ & 0.030 \\
\hline Anorexia & $40(12.4 \%)$ & $15(21.4 \%)$ & $25(9.9 \%)$ & 0.009 \\
\hline Asymptomatic but nucleic acid positive & $24(7.4 \%)$ & $2(2.9 \%)$ & $22(8.7 \%)$ & 0.100 \\
\hline Radiology manifestation & & & & $<0.001$ \\
\hline Unilateral involved & $35(10.8 \%)$ & $3(4.3 \%)$ & $32(12.6 \%)$ & \\
\hline Bilateral involved & $255(79.0 \%)$ & $67(95.7 \%)$ & $188(74.3 \%)$ & \\
\hline Normal & $33(10.2 \%)$ & 0 & $33(13.1 \%)$ & \\
\hline
\end{tabular}

Data are Median (IQR) or $\mathrm{n}(\%), P$ value are calculated by $\mathrm{X}^{2}$ test or Mann-Whitney $\mathrm{U}$ test

and all of those symptoms were significantly more common and frequent in the progressive group. Notably, diarrhea and abdominal discomfort occurred in $7.4 \%$ of the patients and were slightly different in the progressive and stable cohorts (10.0\% vs $6.7 \%)$. As for the vital signs, the progressive group tended to have significantly higher temperature and systolic blood pressure, and prone to tachypnea and low oxygenation index compared to the stable one. Interestingly, 24 patients were asymptomatic on admission but still timely hospitalized due to an exposure history and a laboratory-confirmed positive nucleic acid result of COVID-19 virus.

All patients underwent chest CT on admission, 255 (79.0\%) patients presented bilateral pneumonia and 35 (10.8\%) patients presented unilateral involved, while 33 (10.2\%) patients showed almost no abnormalities. The progressive group displayed more lobes and segments involved, higher proportion of multiple ground-glass opacities, yet all 33 normal CT appeared only in the stable group. 
The laboratory parameters of the progressive and stable patients

As shown in Table 2, there were numerous differences in laboratory findings between the two groups. On admission, the progressive group presented slightly higher white blood cells and neutrophils $(\mathrm{P}=0.026)$ than the stable one. However, the counts of Lymphocytes,
T lymphocytes, CD4+ cell, CD8+ cell and platelets were significantly lower in the progressive patients, resulting in comparatively high level of Neutrophil-to-Lymphocyte Ratio (NLR). Generally, the baseline parameters representing the function of liver (alanine aminotransferase, aspartate aminotransferase, gamma glutamyl transferase), kidney (Creatinine Cr, blood urea nitrogen BUN)

Table 2 Laboratory examination between the progressive and Stable patients

\begin{tabular}{|c|c|c|c|c|c|}
\hline & Normal Range & Total $(\mathrm{N}=323)$ & Progressive $(\mathrm{N}=70)$ & Stable $(\mathrm{N}=253)$ & $P$ value \\
\hline \multicolumn{6}{|c|}{ Blood cell count and lymphocyte classification } \\
\hline White blood Cell $\left(\times 10^{9} / \mathrm{L}\right)$ & $3.5-9.5$ & $4.57(3.55-5.65)$ & $4.39(3.62-5.72)$ & $4.60(3.57-5.63)$ & 0.672 \\
\hline Neutrophils $\left(\times 10^{9} / \mathrm{L}\right)$ & $1.8-6.3$ & $2.56(1.86-3.45)$ & $2.84(2.13-4.19)$ & $2.52(1.79-3.38)$ & 0.026 \\
\hline Lymphocytes $\left(\times 10^{9} / \mathrm{L}\right)$ & $1.1-3.2$ & $1.27(0.99-1.73)$ & $1.02(0.84-1.23)$ & $1.38(1.07-1.85)$ & $<0.001$ \\
\hline NLR & & $1.90(1.28-2.88)$ & $2.72(1.87-4.37)$ & $1.72(1.19-2.53)$ & $<0.001$ \\
\hline Hemoglobin (g/L) & $115-150$ & $136.0(127.0-146.0)$ & $138.0(128.5-148.5)$ & $136.0(127.0-146.0)$ & 0.223 \\
\hline Platelets $\left(10^{9} / \mathrm{L}\right)$ & $125-350$ & $180.0(143.0-224.0)$ & $147.0(122.5-181.0)$ & $190.5(154.0-238.0)$ & $<0.001$ \\
\hline T lymphocyte (N) & $770-2041$ & $980.0(650.0-1317)$ & $529.0(387.0-712.5)$ & $1071(772.5-1399)$ & $<0.001$ \\
\hline CD4 cell (N) & $500-1500$ & $525.0(361.0-714.0)$ & $302.0(204.5-383.0)$ & $596.5(452.5-757.0)$ & $<0.001$ \\
\hline CD8 cell (N) & & $356.0(224.5-515.0)$ & $201.0(134.5-294.0)$ & $402.5(273.0-546.5)$ & $<0.001$ \\
\hline $\mathrm{CD} 4 / \mathrm{CD} 8$ & $1.5-2.5$ & $1.49(1.09-1.96)$ & $1.61(1.02-1.94)$ & $1.48(1.12-1.96)$ & 0.907 \\
\hline \multicolumn{6}{|l|}{ Blood biochemistry } \\
\hline Total bilirubin (uM) & $1.7-21$ & $9.80(7.60-14.6)$ & $10.4(8.10-16.0)$ & $9.55(7.45-14.35)$ & 0.303 \\
\hline $\mathrm{ALT}(\mathrm{U} / \mathrm{L})$ & $<45$ & $20.0(15.0-30.1)$ & $26.0(19.0-38.3)$ & $19.0(13.0-27.0)$ & $<0.001$ \\
\hline AST (U/L) & $<45$ & $26.0(21.0-35.0)$ & $30.0(23.5,42.2)$ & $25.0(20.0-33.0)$ & $<0.001$ \\
\hline GGT (U/L) & $<49$ & $24.0(15.3-39.0)$ & $38.0(23.0-62.0)$ & $21.3(14.5-32.0)$ & $<0.001$ \\
\hline $\mathrm{ALP}(\mathrm{U} / \mathrm{L})$ & $35-100$ & $60.0(50.0-78.0)$ & $59.0(48.9-72.5)$ & $60.5(51.0-82.0)$ & 0.101 \\
\hline Potassium (mM) & $3.5-5.5$ & $3.89(3.62-4.15)$ & $3.80(3.53-4.09)$ & $3.90(3.64-4.17)$ & 0.031 \\
\hline Sodium (mM) & $135-145$ & $138.2(136.5-139.7)$ & $136.2(134.7-139.1)$ & $138.4(137.1-139.8)$ & $<0.001$ \\
\hline BUN (mM) & $2.6-7.5$ & $3.92(3.20-4.92)$ & $4.78(3.66-5.84)$ & $3.80(3.13-4.67)$ & $<0.001$ \\
\hline $\mathrm{Cr}(\mathrm{uM})$ & $41-73$ & $62.0(49.9-75.0)$ & $71.0(61.8-94.0)$ & $58.0(48.0-73.0)$ & $<0.001$ \\
\hline eGFR (ml/min) & $90-250$ & $108.6(96.2-119.7)$ & $94.6(77.0-105.6)$ & $111.2(98.0-121.2)$ & $<0.001$ \\
\hline Troponin T (ug/L) & $<0.012$ & $0.012(0,006-0.012)$ & $0.012(0.012-0.013)$ & $0.012(0.006-0.012)$ & $<0.001$ \\
\hline Creatine kinase & 18.0-198.0 & $69.5(50.0-96.0)$ & $82.5(56.0-126.0)$ & $67.0(48.5-90.5)$ & 0.099 \\
\hline CK-MB (ng/mL) & $0-2.37$ & $0.54(0.22-1.06)$ & $0.62(0.22-1.19)$ & $0.55(0.22-1.07)$ & 0.426 \\
\hline $\mathrm{LDH}(\mathrm{U} / \mathrm{L})$ & 153 & $218.0(174.0-379.0)$ & $283.0(199.0-577.0)$ & $207.0(166.0-323.0)$ & $<0.001$ \\
\hline \multicolumn{6}{|l|}{ Infection-related parameters } \\
\hline $\operatorname{ESR}(s)$ & $0-20$ & $30.0(15.0-49.0)$ & $34.5(26.0-51.0)$ & $23.5(13.0-43.0)$ & $<0.001$ \\
\hline $\mathrm{CRP}(\mathrm{mg} / \mathrm{L})$ & $<8$ & $9.9(3.84-26.2)$ & $26.64(10.4-48.6)$ & $7.25(2.80-19.07)$ & $<0.001$ \\
\hline $\mathrm{PCT}(\mathrm{ng} / \mathrm{mL})$ & $<0.1$ & $0.04(0.03-0.06)$ & $0.06(0.05-0.09)$ & $0.03(0.02-0.05)$ & $<0.001$ \\
\hline IL-6 (pg/mL) & $<7$ & $10.6(4.12-19.7)$ & $22.8(13.1-32.1)$ & $7.77(3.61-14.9)$ & $<0.001$ \\
\hline Oxygenation index $(\mathrm{mmHg})$ & $400-500$ & $420.0(360.5-477.0)$ & 355.5 (296.0-401.0) & $439.0(378.5-495.0)$ & $<0.001$ \\
\hline $\mathrm{PaO}_{2}(\mathrm{mmHg})$ & $75-110$ & $92.3(79.2-106.0)$ & $75.8(69.8-85.9)$ & $97.1(84.4-108.0)$ & $<0.001$ \\
\hline $\mathrm{PCO}_{2}(\mathrm{mmHg})$ & $35-45$ & $39.0(36.1-41.4)$ & $37.2(33.3-39.0)$ & $39.6(37.0-41.9)$ & $<0.001$ \\
\hline \multicolumn{6}{|l|}{ Coagulation function } \\
\hline $\mathrm{PT}(\mathrm{s})$ & $11-15.1$ & $11.8(11.3-12.4)$ & $12.0(11.3-12.6)$ & $11.8(11.2-12.3)$ & 0.088 \\
\hline APTT (s) & $28-43.5$ & $35.3(32.5-38.5)$ & $36.7(34.6-40.2)$ & $34.7(31.8-38.3)$ & 0.002 \\
\hline D-Dimer (s) & $0-0.5$ & $0.36(0.26-0.53)$ & $0.53(0.35-0.64)$ & $0.34(0.25-0.50)$ & $<0.001$ \\
\hline
\end{tabular}

Data are Median (IQR), P value comparing Progressive and Stable group are calculated by Mann-Whitney U test. NLR:Neutrophil-to-Lymphocyte Ratio; ALT, alanine transaminase; AST, aspartate transaminase; GGT, gamma glutamyl transferase; ALP, alkaline phosphatase; CK-MB, creatine kinase-MB; LDH, lactate dehydrogenase; BUN, blood urea nitrogen; $\mathrm{Cr}$, Creatinine; eGFR, estimated Glomerular filtration rate; ESR, Erythrocyte sedimentation rate; CRP, C-reactive protein; PCT, procalcitonin; IL-6, Interleukin 6; PT, prothrombin time; APTT, activated partia I thromboplastin time 
and myocardial zymogram (Troponin T, LDH) were distinctly elevated in the progressive group, indicating the potential organ dysfunction at the beginning. The blood levels of sodium, potassium and $\mathrm{PO}_{2}, \mathrm{PCO}_{2}$, oxygenation index were statistically lower in progressive patients, while elevated level of the infection-related indexes, i.e. ESR, CRP, procalcitonin, interleukin-6 (IL-6) were significantly more prevalent in this group on admission, as with the D-Dimer level. The preliminary results of blood test had already altered visibly in the progressive patients at early stage.

\section{Treatments and outcomes of all 333 patients}

As shown in Table 3, all of the 333 patients, most patients (71.7\%) had oxygen therapy and all patients received antiviral treatment. For severe cases, there was a significantly higher proportion of patients used antibiotics (60.8\%), corticosteroid and gamma globulin (both over $75 \%)$ for treatment compared with the non-severe one. The most frequently used antibiotics were cephalosporin and quinolones. The mainly corticosteroid administrated was Methylprednisolone, the dosage of which was $1-2 \mathrm{mg} / \mathrm{kg} /$ day, maximum used shall be less than 3-5 day. No opportunistic infection was found. All the severe patients had oxygen support. In addition, 23 patients were admitted to intensive care unit, 11 of them had to use the invasive mechanical ventilation and 5 patients switched to extracorporeal membrane oxygenation. The most common complication was acute respiratory distress syndrome (ARDS) which happened to 13 severe patients. Other included acute cardiac injury, acute renal injury, septic shock and multiple organ failure which led to death cases. All 3 death cases were males and over 60 years old, one coexisting with hypertension and another with chronic obstructive pulmonary disease. Two of them were severe-critically ill at admission. Still, more than 240 patients were recovered and discharged from the hospital by February 28th.

\section{The potential risk factors of disease progression}

To predict the risk factors of disease progression based on the clinical features, we found that age, sex, history of exposure, comorbidities, radiology manifestation were significantly associated with the disease progression by the univariate logistic analysis. Furthermore, aged over 40 years, male sex, with comorbidities, a clear and certain exposure history and abnormal radiology manifestations were all risk factors for disease progression by the multivariate logistic analysis (Tables 4).

As shown in the Table 5 of laboratory parameters, the univariate logistic analysis suggested that the baseline levels of NLR, T lymphocyte, BUN, CRP, IL-6, ESR were significantly associated with the disease progression. However, the multivariate logistic analysis indicated that low T lymphocyte level and high levels of CRP, IL-6, NLR were risk factors for disease progression (Table 5).

The diagnosis value and predictors of disease progression Furthermore, through the ROC curve test (Fig. 3), the best cut-off point of age $(\mathrm{AUC}=0.767)$ was 53.5 years, with a specificity of $70 \%$ and a sensitivity of $28.1 \%$. And the ROC curve of T lymphocyte $(\mathrm{AUC}=0.865)$ suggested that the best cut-off point was $825 /$ ul with a specificity of $88.4 \%$ and a sensitivity of $26.3 \%$. The ROC curve of CRP $(\mathrm{AUC}=0.0 .768)$ suggested that the best cut-off point was

Table 3 The treatment between the progressive and stable patients

\begin{tabular}{|c|c|c|c|c|}
\hline & Total $(N=333)$ & $\begin{array}{l}\text { Mild-moderate } \\
(\mathrm{N}=254)\end{array}$ & Severe-critical $(N=79)$ & $P$ value \\
\hline \multicolumn{5}{|l|}{ Treatment } \\
\hline Antiviral therapy & 333 & 254 & 79 & \\
\hline Antibiotic therapy & $99(29.7 \%)$ & $51(20.1 \%)$ & $48(60.8 \%)$ & $<0.001$ \\
\hline Use of corticosteroid & $90(27.0 \%)$ & $22(8.67 \%)$ & $68(86.1 \%)$ & $<0.001$ \\
\hline Use of gamma globulin & $81(24.3 \%)$ & $18(7.1 \%)$ & $63(79.7 \%)$ & $<0.001$ \\
\hline Regulate intestinal flora & $179(53.8 \%)$ & $123(48.4 \%)$ & $56(70.1 \%)$ & $<0.001$ \\
\hline Oxygen support & $238(71.5 \%)$ & $160(63.0 \%)$ & $79(100.0 \%)$ & \\
\hline Nasal cannula & $181(54.4 \%)$ & $157(61.8 \%)$ & $23(29.1 \%)$ & $<0.001$ \\
\hline Mask oxygen inhalation & $7(2.1 \%)$ & $3(1.2 \%)$ & $4(5.1 \%)$ & 0.036 \\
\hline High-flow nasal cannula & $10(3.0 \%)$ & 0 & $10(12.7 \%)$ & \\
\hline Non-invasive ventilation & $24(7.2 \%)$ & 0 & $24(30.4 \%)$ & \\
\hline Invasive mechanical ventilation & $13(3.9 \%)$ & 0 & $13(16.5 \%)$ & \\
\hline Invasive mechanical ventilation + ECOM & $5(1.5 \%)$ & 0 & $5(6.3 \%)$ & \\
\hline Acute respiratory distress syndrome & $13(3.9 \%)$ & 0 & $13(16.5 \%)$ & \\
\hline
\end{tabular}


Table 4 Risk factors of basic information for progression by logistic regression

\begin{tabular}{|c|c|c|c|c|}
\hline \multirow[t]{2}{*}{ Variable } & \multicolumn{2}{|c|}{ Univariate analysis } & \multicolumn{2}{|c|}{ Multivariate analysis } \\
\hline & OR $(95 \% \mathrm{Cl})$ & $P$ value & OR $(95 \% \mathrm{CI})$ & $P$ value \\
\hline Age (> 40 years $v s \leq 40)$ & $7.39(3.52,15.53)$ & $<0.001$ & $3.80(1.72,8.52)$ & 0.001 \\
\hline Sex (male vs. female) & $2.38(1.37,4.11)$ & 0.002 & $2.21(1.20,4.07)$ & 0.011 \\
\hline History of exposure (yes vs no) & $0.249(0.14,0.44)$ & $<0.001$ & $0.38(0.20,0.71)$ & 0.002 \\
\hline Incubation period (days) & $1.01(0.95,1.07)$ & 0.723 & & \\
\hline Comorbidities (yes vs no) & $2.86(1.87,4.38)$ & $<0.001$ & $1.78(1.13,2.81)$ & 0.013 \\
\hline Radiology manifestation (yes vs no) & $5.38(1.89,15.35)$ & 0.002 & $3.56(1.13,11.40)$ & 0.032 \\
\hline Symptoms (yes vs no) & $1.02(0.68,1.53)$ & 0.914 & & \\
\hline Respiratory rate (> 24 breaths/min) & $1.06(0.92,1.21)$ & 0.480 & & \\
\hline
\end{tabular}

Table 5 Risk factors of lab test for progression by logistic reprogression

\begin{tabular}{|c|c|c|c|c|}
\hline \multirow[t]{2}{*}{ Variable } & \multicolumn{2}{|l|}{ Univariate analysis } & \multicolumn{2}{|c|}{ Multivariate analysis } \\
\hline & OR $(95 \% \mathrm{Cl})$ & $P$ value & OR $(95 \% \mathrm{Cl})$ & $P$ value \\
\hline White blood cell $\left(\times 10^{9} / \mathrm{L}\right)$ & $0.97(0.84,1.12)$ & 0.655 & & \\
\hline NLR & $1.44(1.23,1.68)$ & $<0.001$ & $0.99(0.97,1.01)$ & 0.048 \\
\hline T lymphocyte & $0.996(0.994,0.997)$ & $<0.001$ & $0.99(0.997,0.999)$ & 0.002 \\
\hline CD4/CD8 & $1.02(0.68,1.52)$ & 0.939 & & \\
\hline TBIL & $1.02(0.98,1.06)$ & 0.325 & & \\
\hline ALP & $0.99(0.98,1.00)$ & 0.067 & & \\
\hline Troponin T & $0.92(0.60,1.51)$ & 0.738 & & \\
\hline CRP & $1.04(1.02,1.05)$ & $<0.001$ & $1.67(1.12,2.47)$ & 0.012 \\
\hline PCT & $0.90(0.32,2.51)$ & 0.833 & & \\
\hline IL-6 & $1.05(1.03,1.07)$ & $<0.001$ & $1.03(1.00,1.05)$ & 0.008 \\
\hline ESR & $1.02(1.00-1.03)$ & 0.002 & $0.99(0.97,1.01)$ & 0.529 \\
\hline D-Dimer & $1.78(1.02,3.09)$ & 0.052 & & \\
\hline Ferritin & $1.00(1.000-1.001)$ & 0.189 & & \\
\hline
\end{tabular}

NLR, Neutrophil-to-Lymphocyte Ratio; CRP, C-reactive protein; PCT, procalcitonin
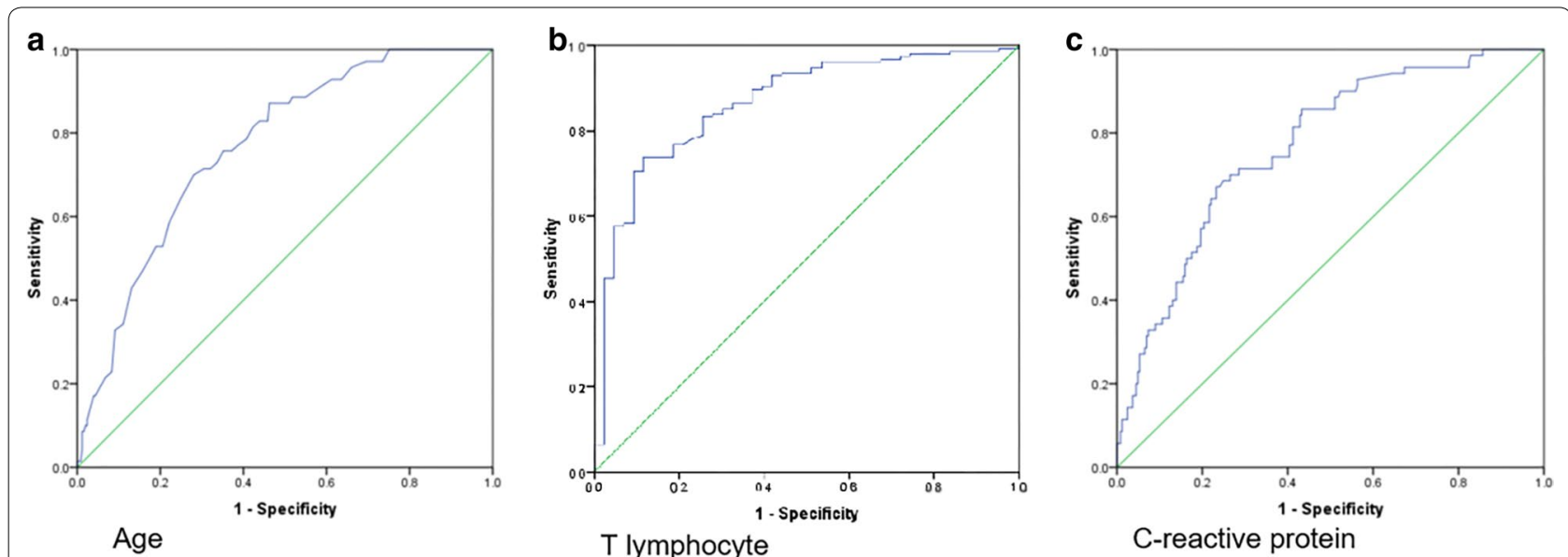

Fig. 3 The ROC curve of age, T lymphocyte and CRP of the progressive and stable patients. a ROC curve of age; b ROC curve of T lymphocyte; $\mathbf{C}$ $\mathrm{ROC}$ curve of $\mathrm{C}$-reactive protein 
$9.71 \mathrm{mg} / \mathrm{ml}$ with a specificity of $81.4 \%$ and a sensitivity of $41.2 \%$.

Compared with the stable group, the length of disease progressing time was significantly different according to the age and sex by the Kaplan-Meier analysis (Fig. 4). It can be inferred that the elderly and male patients were more likely to progress into severe-critically ill conditions.

\section{Discussion}

Since the rapid person-to-person transmission of COVID-19 outbreak occurred in December 2019, the number of infected cases had risen exponentially. The world was on the brink of a pandemic [9, 10]. In our study, we retrospectively assessed the clinical characteristics and medical tests of 333 patients infected with COVID-19 in the only designated hospital in Shenzhen and tried to analyze and identify the baseline risk factors for clinical progression.

COVID-19 appeared to pose a particular threat to middle-aged and older adults, especially men, while it spared the underage group. None of the patients under 18 years (35 minors, $10.5 \%$ ) deteriorated and all remained a mild-moderate condition. However, aged over 40 years had taken up a prominently high proportion (over 87\%) of the progressive group, while patients under 40 years accounted for more than half of the stable group. Although men and women were infected in roughly equal numbers, the number of males in severe-critically ill condition was almost twice as compared to women (52 vs
27). Besides, all three death cases were male. Underling disease was another contributing factor. Nearly half of the severe cases were coexisting with at least one comorbidity. Patients with two or more comorbidities had a significantly elevated risk of exacerbation [11]. We also noticed that among all the COVID-19 infections, there were 4 patients infected with respiratory syncytial virus and 2 patients with influenza B virus concurrently, which did not result in aggravated condition but remained mild and moderate. In the early stage of the transmission, we found most infected cases were directly related to Wuhan or Hubei province and only $3.4 \%$ patients claimed unclear exposure history, which meant we can easily trace the source of virus transmission and isolate the suspected ones. Furthermore, patients who had an early onset and admitted to hospital before January 25th tended to be more severe and progressive, for 19 out of 48 patients (40\%) developed to severe-critically ill conditions and two of them even died.

Until February 28th, more than 240 patients were recovered in our study, 62 (18.7\%) of covid-19 infections were diagnosed as severe cases and 17 patients (5.1\%) were critically ill, while the mortality rate was estimated $0.9 \%$, much lower than Hubei region had reported [12]. Fever was the most universal symptom among all, but patients can be afebrile and respiratory symptoms were not presented in all cases. Unlike a common cold, sore throats and rhinorrhea or rhinobyon were relatively rare. Diarrhea might be underestimated since ACE2 was highly expressed in the small intestine which can be
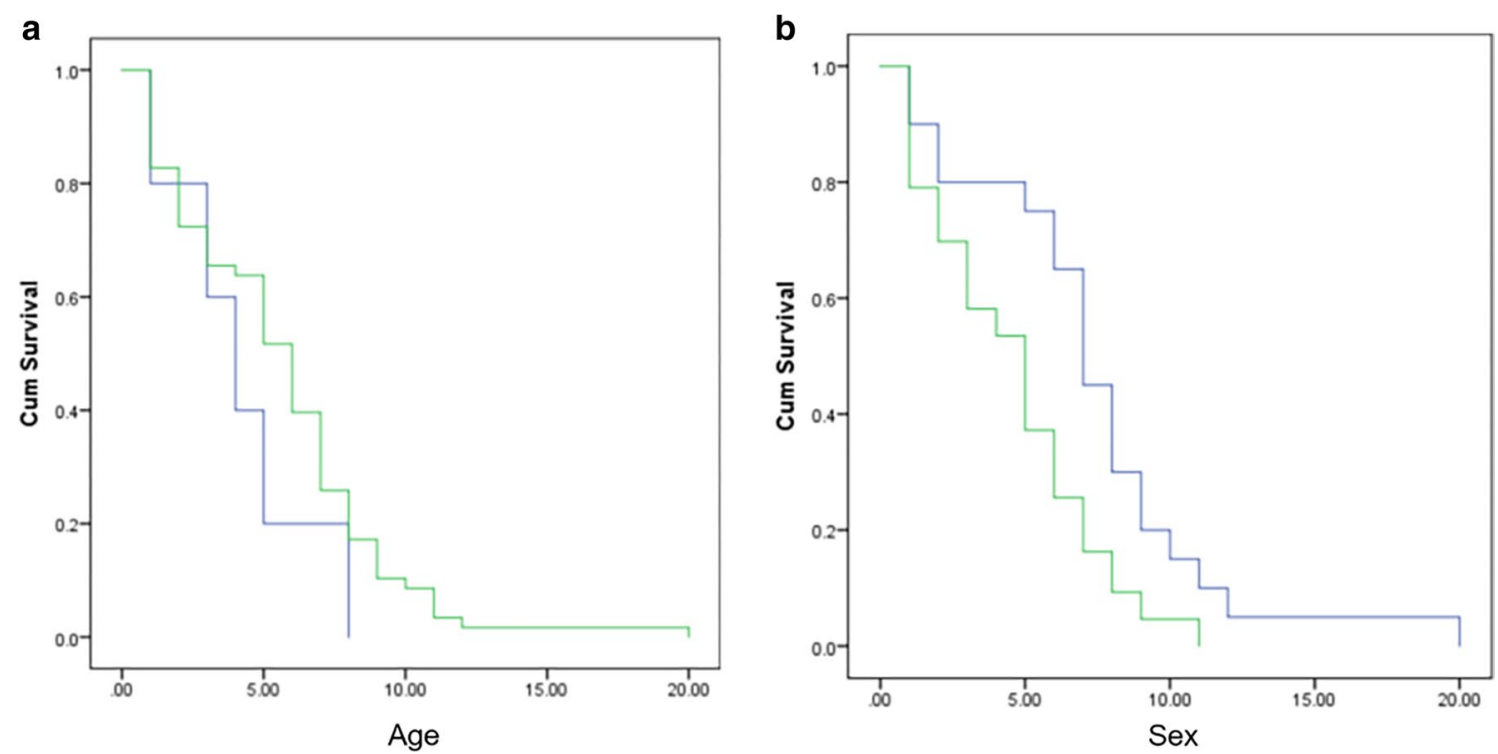

Fig. 4 Comparison of the interval of disease progression between the progressive and stable patients. The interval of disease progression by age (a) and sex (b) by the Kaplan-Meier analysis 
attacked by the virus [13]. Therefore, we should be cautious when a feverish patient with diarrhea saw a doctor. In terms of severe cases, high fever with systemic symptoms may be predictive for clinical progression [14]. All three death cases had multiple symptoms including fever, cough, dyspnea, fatigue, anorexia at admission and then developed into ARDS. In addition, a small proportion were asymptomatic patients screened out from the nucleic acid test and close contacts. It can be contagious as well and may threaten the life of other cohabiting members, especially the elderly ones. Notably, among the 24 carriers, 12 patients aged under 18 years and showed only mild symptoms during hospitalization. 15 patients had already shown the radiologic feature of COVID-19 pneumonia in chest CT scan upon admission. Only 2 of them progressed to severe while 22 remained stable and mild, which indicated the importance of continuous nucleic acid tests and early abnormalities detection in CT imaging $[8,15,16]$. Diagnosis in the early phase and isolated for medical observation may be helpful to the whole community.

In terms of laboratory test results, lymphocytes, especially $\mathrm{T}$ lymphocytes were significantly reduced in severe cases, which indicates COVID-19 consumed immune cells and inhibits the cellular immune function. In addition, progressive patients tend to have higher baseline NLR, ESR, CRP, IL-6, D-dimer level, which may be related to inflammatory response and cytokine storm induced by virus invasion $[17,18]$. Our results were also in line with other retrospective studies $[19,20]$. Those inflammatory factors level at admission may help to identify and determine later clinical progression. A higher level suggested more significant risk to exacerbation. Nearly all the patients received antiviral treatment. Lopinavir/ritonavir were reported to have potentially therapeutic effects on SARS and widely applied [21]. Antibiotics were strictly controlled and only prescribed to patients with a highly suspected bacterial infection in our hospital. Corticosteroid and gamma globulin were typically used in severe cases to reduce lung inflammatory response.

The reason for the rapid expansion might be associated with the mild and atypical symptoms in the early stage of infected individuals. As there were no specific and effective antiviral therapies identified, our suggestion was to control the source of infection, as well as the use of facial mask for protection, isolation and early diagnosis. More importantly, early identification of risk factors associated with the clinical progression of COVID-19 should be paid prominent attention for better patient management and stratification.

The study is subjected to certain limitations. Firstly, as the epidemics has not ended yet and many patients are still hospitalized at the time of study submission, we are unable to estimate the overall proportion of clinical progression and case fatality rate. Secondly, due to the retrospective nature of the study, a systematic selection bias and residual confounding factors cannot be fully addressed and may lead to inaccurate conclusion. Thirdly, the clinical predictive value remains to be explored and a multi-center and follow-up study with a larger cohort is highly required.

\section{Conclusion}

In this single-center case series of 333 hospitalized patients with confirmed COVID-19 in Shenzhen, China, we assessed and analyzed the clinical characteristics and potential predictors of disease progression and prognosis on admission and found the risk factors of males, older age, with comorbidities, low $\mathrm{T}$ lymphocyte level and high level of NLR, CRP, IL-6 can help to predict clinical progression of COVID-19 at an early stage.

\section{Acknowledgements}

The authors would like to show their respect for all the hospital staff for their hard work and efforts to combat the COVID-2019.

\section{Authors' contributions}

$\mathrm{LL}$ and $\mathrm{QH}$ had full access to the data and take responsibility for the accuracy of the data. FW, MQ, XZ and KZ contributed equally and share first authorship. Data acquisition: FW, CL, PH, QT, WX, RC, XL, ZL; data analyses and interpretation: FW, MQ, KZ. Concept and design: KZ, LL, QH; Manuscript drafting: MQ, $X Z$ and $K Z$; Critical revision of the manuscript: FW, LL, QH. All authors read and approved the final manuscrip.

\section{Funding}

This work is supported by the National Clinical Research Center for Infectious Diseases, Funds for the construction of key medical disciplines in Shenzhen and the Sanming Project of Medicine in Shenzhen (SZSM201612014).

\section{Availability of data and materials}

The data supporting the conclusions of this article are included within the article.

\section{Ethics approval and consent to participate}

This study was approved by the Ethics Committee of The third people's Hospital of Shenzhen. Due to the urgent exploration for this disease, written informed consent was waived.

\section{Consent for publication}

The manuscript is approved by all authors for publication. This article does not contain any individual person's data in any form.

\section{Competing interests}

No conflict of interest exits in the manuscript.

\section{Author details}

${ }^{1}$ Department of Hepatology, Shenzhen Third People's Hospital, National

Clinical Research Center for Infectious Disease, The Second Affiliated Hospital, School of Medicine, Southern University of Science and Technology, 29 Bulan Road, Shenzhen 518112, Guangdong, China. ${ }^{2}$ Institute of Reproductive Health, Tongji Medical College, Huazhong University of Science and Technology, Wuhan, China.

Received: 5 May 2020 Accepted: 17 June 2020

Published online: 03 July 2020 


\section{References}

1. Zhu N, Zhang D, Wang W, et al. A novel coronavirus from patients with pneumonia in China, 2019. N Engl J Med. 2020;382(8):727-33.

2. Huang C, Wang Y, Li X, et al. Clinical features of patients infected with 2019 novel coronavirus in Wuhan, China. Lancet. 2020;395(10223):497-506

3. Fauci AS, Lane HC, Redfield RR. Covid-19-navigating the uncharted. J Med. 2020;382:1268-69.

4. Wang D, Hu B, Hu C, et al. Clinical characteristics of 138 hospitalized patients with 2019 novel coronavirus-infected pneumonia in Wuhan, China. Jama. 2020;323(11):1061-69.

5. $X u X W, W u X X$, Jiang $X G$, et al. Clinical findings in a group of patients infected with the 2019 novel coronavirus (SARS-Cov-2) outside of Wuhan, China: retrospective case series. BMJ. 2020;368:m606.

6. Yang $X, Y u Y, X u$ J, et al. Clinical course and outcomes of critically ill patients with SARS-CoV-2 pneumonia in Wuhan, China: a singlecentered, retrospective, observational study. Lancet Respir Med. 2020;8(5):475-81.

7. WHO. Clinical management of severe acute respiratory infection when novel coronavirus (2019-nCoV) infection is suspected: interim guidance, 28 January 2020. Geneva: World Health Organization; 2020.

8. Chung M, Bernheim A, Mei X, Zhang N, Huang M, Zeng X, Cui J, Xu W, Yang Y, Fayad ZA, Jacobi A. CT imaging features of 2019 novel coronavirus (2019-nCoV). Radiology. 2020;295(1):202-7.

9. Day M. Covid-19: surge in cases in Italy and South Korea makes pandemic look more likely. BMJ. 2020;368:m751.

10. Cowling BJ, Leung GM. Epidemiological research priorities for public health control of the ongoing global novel coronavirus (2019-nCoV) outbreak. Euro Surveill. 2020;25(6):2000110.

11. Guan W, Liang W, Zhao Y, et al. Comorbidity and its impact on 1,590 patients with COVID-19 in China: A Nationwide Analysis. medRxiv 2020:2020-2.

12. Chen N, Zhou M, Dong X, et al. Epidemiological and clinical characteristics of 99 cases of 2019 novel coronavirus pneumonia in Wuhan, China: a descriptive study. Lancet. 2020;395(10223):507-13.
13. Liang W, Feng Z, Rao S, Xiao C, Xue X, Lin Z, Zhang Q, Qi W. Diarrhoea may be underestimated: a missing link in 2019 novel coronavirus. Gut. 2020;69(6):1141-43.

14. Wu Z, McGoogan JM. Characteristics of and important lessons from the coronavirus disease 2019 (COVID-19) outbreak in China: summary of a report of 72314 cases from the Chinese Center for Disease Control and Prevention. JAMA. 2020;323(13):1239-42.

15. Bai Y, Yao L, Wei T, et al. Presumed asymptomatic carrier transmission of COVID-19. JAMA. 2020;323(14):1406-7.

16. Shi $H$, Han $X$, Jiang $N$, et al. Radiological findings from 81 patients with COVID-19 pneumonia in Wuhan, China: a descriptive study. Lancet Infect Dis. 2020;20:425-34.

17. Gong J, Dong H, Xia SQ, et al. Correlation Analysis Between Disease Severity and Inflammation-related Parameters in Patients with COVID-19 Pneumonia. medRxiv 2020:2020-2.

18. Chen G, Wu D, Guo W, et al. Clinical and immunologic features in severe and moderate forms of Coronavirus Disease 2019. medRxiv 2020 2020-2.

19. Liu J, Li S, Liu J, et al. Longitudinal characteristics of lymphocyte responses and cytokine profiles in the peripheral blood of SARS-CoV-2 infected patients. Ebiomedicine. 2020:55:102763.

20. Qin C, Zhou L, Hu Z, et al. Dysregulation of immune response in patients with COVID-19 in Wuhan, China. Clin Infect Dis. 2020. https://doi. org/10.1093/cid/ciaa248.

21. Chu CM, Cheng VC, Hung IF, et al. Role of lopinavir/ritonavir in the treatment of SARS: initial virological and clinical findings. Thorax. 2004;59(3):252-6.

\section{Publisher's Note}

Springer Nature remains neutral with regard to jurisdictional claims in published maps and institutional affiliations.
Ready to submit your research? Choose BMC and benefit from:

- fast, convenient online submission

- thorough peer review by experienced researchers in your field

- rapid publication on acceptance

- support for research data, including large and complex data types

- gold Open Access which fosters wider collaboration and increased citations

- maximum visibility for your research: over $100 \mathrm{M}$ website views per year

At BMC, research is always in progress.

Learn more biomedcentral.com/submissions 